\title{
Medical Student Medium-Term Skill Retention Following Cardiac Point-of-Care Ultrasound Training Based on the American Society of Echocardiography Curriculum Framework
}

\section{Satoshi Jujo ( $\nabla$ neo.j.j@live.jp )}

University of Hawai'i at Manoa John A Burns School of Medicine https://orcid.org/0000-0003-1933-

0782

\section{Brandan I Sakka}

University of Hawai'i at Manoa John A Burns School of Medicine Jannet $\mathbf{J}$ Lee-Jayaram

University of Hawai'i at Manoa John A Burns School of Medicine

\section{Akihisa Kataoka}

Teikyo University: Teikyo Daigaku

Masaki Izumo

St Marianna University School of Medicine: Sei Marianna Ika Daigaku

\section{Kenya Kusunose}

Tokushima University Hospital: Tokushima Daigaku Byoin

\section{Atsushi Nakahira}

University of Hawai'i at Manoa John A Burns School of Medicine

\section{Sayaka Oikawa}

Fukushima Medical University: Fukushima Kenritsu Ika Daigaku

\section{Yuki Kataoka}

Min-Iren Asukai Hospital, Kyoto, Japan

\section{Benjamin W Berg}

University of Hawai'i at Manoa John A Burns School of Medicine

\section{Research Article}

Keywords: Point-of-care ultrasound, Handheld ultrasound, Medical education, Medical student, Skill retention

Posted Date: February 28th, 2022

DOI: https://doi.org/10.21203/rs.3.rs-1362150/v1 
License: (c) (i) This work is licensed under a Creative Commons Attribution 4.0 International License. Read Full License

Version of Record: A version of this preprint was published at Cardiovascular Ultrasound on October 12th, 2022. See the published version at https://doi.org/10.1186/s12947-022-00296-z. 


\section{Abstract \\ Background}

No studies have demonstrated medium- or long-term skill retention of cardiac point-of-care ultrasound (POCUS) curriculum for medical student. Based on the American Society of Echocardiography (ASE) curriculum framework, we developed a blended-learning cardiac POCUS curriculum with competency evaluation. The objective of this study was to investigate the curriculum impact on image acquisition skill retention 8 weeks after initial training.

\section{Methods}

This study was a prospective, pre-post education intervention study for first- and second-year medical students, with blinded outcome assessment. The curriculum included a pre-training ASE online module and healthy volunteer hands-on training to obtain 5 views: parasternal long-axis (PLAX), parasternal short-axis (PSAX), apical 4-chamber (A4C), subcostal 4-chamber (S4C), and subcostal inferior vena cava (SIVC) views. Students took 5-view image acquisition skill tests at pre-, immediate post-, and 8-week posttraining, using a healthy volunteer. Three blinded assessors rated the image quality using a validated 10point maximum scoring system. Students used a hand-held ultrasound probe (Butterfly iQ).

\section{Results}

Fifty-four students completed hands-on training, and pre- and immediate post-training skill tests. Twentyseven students completed 8-week post-training skill tests. Skill test score improvement between pre- and 8-week post-training was 2.11 points $(95 \% \mathrm{Cl}, 1.22-3.00$; effect size, 1.13$)$.

\section{Conclusion}

The cardiac POCUS curriculum demonstrated medium-term skill retention. Instructional design modifications or re-training for PLAX, PSAX, and A4C are needed to make the curriculum more effective for clinically relevant skill retention.

\section{Introduction}

Cardiac point-of-care ultrasound (POCUS) is a rapid, bedside cardiac ultrasound examination that assesses important cardiovascular pathology. It is increasingly used in clinical practice by multiple specialties including internal medicine, emergency medicine, critical care medicine, and anesthesiology [1-5]. The development of affordable handheld ultrasound (HHU) devices that operate with smartphones or tablets has further increased the utilization of cardiac POCUS $[5,6]$. Cardiac POCUS is becoming an essential skill for medical students to learn in preparation for their future clinical practice [7]. 
Stethoscopes for medical students could be replaced with HHU or "ultrasound stethoscopes" in the foreseeable future to learn cardiovascular clinical examinations [8].

A review on cardiac POCUS education in medical schools included studies from 12 medical schools in the United States and 6 other countries and demonstrated benefits of cardiac POCUS curricular integration [7]. However, instructional designs of reported curricula are highly variable, lacking standardized methodology or competency evaluation with validity evidence [7]. Moreover, competency evaluation in these studies focused on very short-term skill and/or knowledge retention immediately after an initial training, but provided no insights into medium- or long-term retention $[9,10,19,11-18]$. Developing a curriculum that places greater emphasis on the longevity and durability of a learned skill, rather than immediate recollection or improvement, is important for achieving efficient student learning and effective use of limited instructor time.

To address these issues, the American Society of Echocardiography (ASE) proposed a cardiac POCUS teaching framework for medical students [7]. The ASE framework includes a pre-training didactic education with e-learning (https://aselearninghub.org/), hands-on training, and a competency evaluation. The training goals include enhancing cardiac physical examination skills and augmenting learning of normal anatomy, rather than learning advanced pathology. In our pilot study with 6 pre-clinical medical students, the cardiac POCUS curriculum with the ASE framework demonstrated that students improved image acquisition skills immediately after training, and improved skills were retained 8 weeks after training with a large effect size (ES) [20]. The pilot study confirmed curriculum feasibility and provided rationale for conducting a full-scale study to statistically confirm the skill retention.

The objective of this study was to elucidate learning effects of the curriculum on medium-term skill retention in pre-clinical medical students for future curriculum utilization. We hypothesized that preclinical medical students would retain improved cardiac POCUS image acquisition skills 8 weeks after initial training.

\section{Methods}

\section{Design}

This was a prospective, single-group, pre-post educational intervention study with blinded outcome assessment.

\section{Participants and Setting}

First- and second-year medical students who had completed the 12-week pre-clinical cardiovascular and pulmonary core curriculum at John A. Burns School of Medicine (JABSOM), University of Hawaii, USA were eligible for the study. We recruited participants through e-mail and public postings. This study was conducted at the JABSOM SimTiki Simulation Center (SimTiki) between September 2019 and June 2020. The University of Hawaii Human Studies Program approved the study (Protocol number: 2019 - 00265). 
All participants provided informed consent, and all data were de-identified after collection. No incentives or reimbursements were provided to participants. We carried out this study in accordance with The Code of Ethics of the World Medical Association (Declaration of Helsinki).

\section{Cardiac POCUS Curriculum}

In our previous pilot study, we developed a basic cardiac POCUS curriculum for pre-clinical medical students based on the ASE-recommended framework that encourages the use of a flipped classroom/blended-learning model with online modules [20]. Student goals for this curriculum were to independently obtain basic cardiac POCUS views in a healthy volunteer and to identify normal anatomic structures seen in cardiac POCUS views. Concepts of curriculum design were underpinned by educational principles for effective learning and skill retention, which include concurrent feedback, deliberate practice, mastery learning, and range of difficulty [21]. Curriculum developers were echocardiography subject matter experts including a Fellow of the European Society of Cardiology (KA), a Fellow of the American Society of Echocardiography (KK), and experienced simulation curriculum developers (BWB and JJL). The curriculum timeline is shown in Fig. 1. The cardiac POCUS curriculum included a pre-training selfstudy of the ASE cardiac POCUS online module and a hands-on training session with a healthy volunteer. The students used an HHU probe (Butterfly iQ; Butterfly Network, Inc., Guilford, CT, USA) with a 9.7-inch tablet display during the training. Student image acquisition skill and anatomical knowledge were assessed before, immediately after, and 8 weeks after training.

ASE cardiac POCUS online module for medical students: The ASE POCUS task force has a free cardiac POCUS online module for medical students (https://aselearninghub.org/). We utilized the ASE online module titled "Cardiovascular Point-of-Care Imaging for the Medical Student and Novice User" as the pretraining didactic. The complete ASE online module comprised 8 sub-modules: Introduction, Basic Anatomy Correlating to Cardiac POCUS Views (module A), Complete Cardiac POCUS Scan (module B), Integrated Cardiac Point-of-Care and Physical Exam (module C), Pathology-I (module D), Pathology-II (module D), Teaching the Teacher (module E), and Standards and Testing (module F). Our pre-training self-study curriculum included the first 4 ASE modules on normal anatomy and physiology (Introduction, modules $A, B$, and C), which were matched to the learner level of pre-clinical medical students without extensive prior knowledge of cardiac pathology. The 4 ASE modules were designed to be completed in approximately $35 \mathrm{~min}$. Students independently reviewed the online modules 1 day to 1 week before hands-on training.

5 cardiac POCUS views selection: We selected 5 cardiac POCUS views for hands-on training: parasternal long-axis (PLAX), papillary muscle level of parasternal short-axis (PSAX), apical 4-chamber (A4C), subcostal 4-chamber (S4C), and subcostal inferior vena cava (SIVC) views. The 5-view selection was based on recommendations by the World Interactive Network Focused on Critical Ultrasound [2], European Association of Cardiovascular Imaging [22], and ASE [5].

Cardiac POCUS hands-on training session: One instructor (SJ) delivered a 30-min interactive 1-on-1 lecture using PowerPoint slides of the ASE online module and a life-size model heart (Cardiac POCUS 
lecture). Content of the lecture is in Additional File 1, and a pre-recorded video of 5-view image acquisition instruction in the lecture is in Additional File 2 (https://youtu.be/3PfRzsYjKQg) (The video is a short edited version of the actual video for this article.). Following lecture, students engaged in a supervised, 1on-1 hands-on training of the 5-view image acquisition on a thin, healthy male volunteer for $30 \mathrm{~min}$ (Cardiac POCUS hands-on training). The instructor assumed the role of the healthy volunteer during the hands-on training while providing concurrent, verbal, and tactile feedback to guide student skill development. During hands-on training, students deliberately practiced until they obtained each image with clinically acceptable quality. Image acquisition instruction was designed with reference to an imaging protocol in the ASE comprehensive transthoracic echocardiography guidelines and a point-ofcare ultrasound textbook $[23,24]$. The main instruction points for the 5 -view image acquisition are presented in Additional File 3.

\section{Skill Test Scoring System}

Skill test: We assessed image acquisition skill at pre-, immediate post-, and 8-week post-training, using a 10-point maximum skill test scoring system. The skill test is demonstrated in Additional File 4 (https://youtu.be/9KOO_vdNf-c) (One of authors, JJL, played the role of a student in the video). During the skill test, students demonstrated the 5 cardiac POCUS views on the same single healthy volunteer as in the hands-on training without guidance. Students were given 2 min to obtain each view, for a total of 10 min for 5 views. Once the students found their "best" view, they pressed the record button on the tablet for a 5 -second clip. Students were allowed to record a maximum of 2 clips for each view. If they had 2 recordings, they selected a single recording for evaluation. We utilized the Butterfly iQ application predefined cardiac ultrasound preset for gain and other ultrasound imaging parameters [25]. We preset the imaging depth to $16 \mathrm{~cm}$ for PLAX and PSAX, $18 \mathrm{~cm}$ for A4C, and $20 \mathrm{~cm}$ for S4C and SIVC. The healthy volunteer was in the left decubitus position for PLAX, PSAX, and A4C, and the supine position with bent knees for S4C and SIVC. The healthy volunteer controlled his respiratory rate at 6 per min and held his breath for 5 seconds when the view recording started.

10-point maximum skill test scoring system: We developed a 10-point maximum scoring system by modifying an existing assessment tool for transthoracic echocardiography views in our previous pilot study $[20,26]$. The scoring system was designed to assess the 5-view image quality for rapid bedside cardiac assessment, not for a formal diagnostic comprehensive echocardiography examination. The 10point maximum skill test scoring system rated the 5 views; each received a score ranging from 0 to 2 points (Table 1). Each view was assessed as excellent (2 points), acceptable (1 point), or poor (0 point) for cardiac POCUS use. The scores from 5 views were summed for a 10-point maximum test score. Excellent quality reference images and videos of the 5 views obtained by a cardiologist (MI) on the healthy volunteer are in Fig. 2A and Additional File 5 (https://youtu.be/DrPp2C7ET8c). Examples of acceptable and poor quality images and videos obtained by participants are in Figs. 2B, 2C, and Additional File 6, 7 (https://youtu.be/fCuYUNW87XY, https://youtu.be/25wj2ml51Pk), respectively. After de-identifying skill test clips including information of pre-, immediate post-, and 8-week post-training, we downloaded the de-identified clips in an electronic database and arranged the clips in randomized order 
using the random number table in Microsoft Excel for blinded assessment. Three independent blinded raters scored the image quality using the scoring system, and the average of the scores from the 3 raters was then utilized as a representative score. The 3 raters were echocardiography experts. In our pilot study, the skill test scoring system demonstrated excellent interrater reliability and test-retest reliability of the 3 raters [20]. It also demonstrated outstanding discriminatory ability between novices and experts for echocardiography in a validation study using skill test scores from 60 medical students in our pilot study and the current study (Additional File 8). 
Table 1

10-point maximum skill test scoring system

\section{$5 \quad$ Points Image quality criteria \\ cardiac \\ POCUS \\ views}

\begin{tabular}{ccl} 
PLAX 2 & Excellent: & $\begin{array}{l}\text { All } 7 \text { chambers and anatomical structures (LA, LV, LVOT, RV, AV, } \\
\text { MV, and IVS) visualized or similar to the excellent quality } \\
\text { reference*. }\end{array}$ \\
\hline 1 & Acceptable: & $\begin{array}{l}\text { One chamber (LA, LV, or RV) severely foreshortened or 1 } \\
\text { anatomical structure (LVOT, AV, MV, or IVS) not visualized well. }\end{array}$ \\
\hline 0 & Poor: & $\begin{array}{l}\text { Any } 2 \text { chambers or structures (LA, LV, LVOT, RV, AV, MV, and IVS) } \\
\text { severely foreshortened/not visualized well, the left and right sides } \\
\text { of the image are flipped, raters do not recognize the view as a } \\
\text { parasternal long-axis view, or no image obtained. }\end{array}$
\end{tabular}

$\begin{array}{llll}\text { PSAX } & 2 & \text { Excellent: } & \text { All } 4 \text { chambers and anatomical structures (round LV, RV, papillary }\end{array}$ muscles, and IVS) visualized or similar to the excellent quality reference*.

$1 \quad$ Acceptable: One chamber or anatomical structure (round LV, RV, papillary muscles, or IVS) not visualized well, oval LV, significant lateral wall drop out of LV compared with the excellent quality reference*, or mitral level of parasternal short-axis view.

0 Poor: $\quad$ Any 2 chambers or anatomical structures (round LV, RV, papillary muscles, and IVS) not visualized well, apical level or aortic valve level of parasternal short-axis view, the left and right sides of the image are flipped, raters do not recognize the view as a parasternal short-axis view, or no image obtained.

\begin{tabular}{ccc} 
A4C 2 Excellent: & $\begin{array}{l}\text { All } 8 \text { chambers and anatomical structures (LA, LV, RA, RV, MV, TV, } \\
\text { IAS, and IVS) visualized or similar to the excellent quality } \\
\text { reference*. }\end{array}$ \\
\hline $1 \quad$ Acceptable: & $\begin{array}{l}\text { One chamber (LA, LV, RA, or RV) severely foreshortened, } 1 \\
\text { anatomical structure (MV, TV, IAS, or IVS) not visualized well, } \\
\text { aortic outflow added (5-chamber view), or significant lateral wall } \\
\text { drop out of LV compared with the excellent quality reference*. }\end{array}$ \\
\hline 0 & Poor: & $\begin{array}{l}\text { Any } 2 \text { chambers or anatomical structures (LA, LV, RA, RV, MV, TV, } \\
\text { IAS, and IVS) not visualized well, the left and right sides of the } \\
\text { image are flipped, raters do not recognize the view as an apical 4- } \\
\text { chamber view, or no image obtained. }\end{array}$
\end{tabular}

S4C 2 Excellent: All 7 chambers and anatomical structures (LA, LV, RA, RV, IAS, IVS, and liver) visualized or similar to the excellent quality reference*.

The left and right side flipped image does not affect the subcostal 4-chamber view scoring.

1 Acceptable:

One chamber or anatomical structure (LA, LV, RA, RV, IAS, IVS, or liver) severely foreshortened/not visualized well or aortic outflow added (5-chamber view). 


\begin{tabular}{|c|c|c|c|}
\hline \multirow{2}{*}{$\begin{array}{l}5 \\
\text { cardiac } \\
\text { POCUS } \\
\text { views }\end{array}$} & Points & \multicolumn{2}{|c|}{ Image quality criteria } \\
\hline & 0 & Poor: & $\begin{array}{l}\text { Any } 2 \text { chambers or anatomical structures (LA, LV, RA, RV, IAS, IVS, } \\
\text { and liver) not visualized well, raters do not recognize the view as a } \\
\text { subcostal } 4 \text {-chamber view, or no image obtained. }\end{array}$ \\
\hline \multirow[t]{3}{*}{ SIVC } & 2 & Excellent: & $\begin{array}{l}\text { IVC visualized in a longitudinal fashion, connection of IVC to RA } \\
\text { visualized clearly, and IVC diameter }>=1.0 \mathrm{~cm} \text { at } 2 \mathrm{~cm} \text { from the } \\
\text { RA-IVC junction, or similar to the excellent quality reference*. The } \\
\text { left and right sides flipped image does not affect the subcostal } \\
\text { IVC view scoring. }\end{array}$ \\
\hline & 1 & Acceptable: & $\begin{array}{l}\text { IVC diameter }>=1.0 \mathrm{~cm} \text { at } 2 \mathrm{~cm} \text { from the RA-IVC junction, but no } \\
\text { clear connection of IVC to RA, or IVC not visualized in a } \\
\text { longitudinal fashion. }\end{array}$ \\
\hline & 0 & Poor: & $\begin{array}{l}\text { IVC diameter }<1.0 \mathrm{~cm} \text { at } 2 \mathrm{~cm} \text { from the RA-IVC junction, } \\
\text { descending aorta imaged instead of IVC, raters do not recognize } \\
\text { the view as a subcostal IVC view, or no image obtained. }\end{array}$ \\
\hline \multicolumn{4}{|c|}{$\begin{array}{l}A V \text {, aortic valve; } A 4 C \text {, apical 4-chamber view; IAS, interatrial septum; } I V C \text {, inferior vena cava; } I V S \text {, } \\
\text { interventricular septum; } L A \text {, left atrium; } L V \text {, left ventricle; } L V O T \text {, left ventricle outflow tract; } M V \text {, mitral } \\
\text { valve; } P L A X \text {, parasternal long-axis view; } P O C U S \text {, point-of-care ultrasound; } P S A X \text {, papillary muscle level } \\
\text { of parasternal short-axis view; } R A \text {, right atrium; } R V \text {, right ventricle; } S I V C \text {, subcostal inferior vena cava } \\
\text { view; } S 4 C \text {, subcostal 4-chamber view; } T V \text {, tricuspid valve. }\end{array}$} \\
\hline \multicolumn{4}{|c|}{$\begin{array}{l}\text { The 2-point maximum scores for each of the } 5 \text { cardiac POCUS views are added for the } 10 \text {-point } \\
\text { maximum skill test score. }\end{array}$} \\
\hline \multicolumn{4}{|c|}{$\begin{array}{l}\text { *Excellent quality reference refers to an image obtained by the cardiologist (MI) on the healthy } \\
\text { volunteer used for all skill tests (Fig. } 2 \text { A and Additional File 5). Adapted from Jujo et al.[20] }\end{array}$} \\
\hline
\end{tabular}

\section{Knowledge Test Scoring System}

We assessed the anatomical knowledge of students before, immediately after, and 8 weeks after training, using an identical knowledge test on Google Forms (Fig. 1). The knowledge test consisted of 40 multiplechoice questions identifying normal anatomic structures seen in the 5 cardiac POCUS views. The 40-point maximum knowledge test scoring system is in Additional File 9. This scoring system demonstrated outstanding discriminatory ability between novices and experts for echocardiography in a validation study using knowledge test scores from 59 medical students in our pilot study and the current study (Additional File 10).

\section{Outcome Measures}

We measured the following curriculum learning effect outcomes: The primary outcome was [ ] and secondary outcomes were [ ]-[ ].

\section{Skill test score improvement}


[ ] skill test score difference between pre-training and 8-week post-training and [ ] the difference between pre-training and immediate post-training.

\section{Knowledge test score improvement}

[ ] knowledge test score difference between pre-training and 8-week post-training and [ ] the difference between pre-training and immediate post-training.

\section{5-point Likert scale questionnaire}

We administered 5-point Likert scale questionnaires using Google Forms to measure [ ] overall curriculum satisfaction, [ ] the ASE online module satisfaction, and [ ] hands-on training satisfaction at immediate post- and 8-week post-training. Questionnaires also assessed [ ] student motivation to purchase a personal HHU at pre-, immediate post-, and 8-week post-training.

\section{Subgroup analysis}

Based on our pilot study findings of individual skill retention variation [20], we planned to perform subgroup analyses to investigate factors that affected skill retention. When we found significant skill test score variation at 8-week post-training by visual inspection, we examined demographic factors between students with a skill test score of 5 or higher and less than 5 at 8-week post-training to investigate the reason for score variation.

\section{Interrater Reliability of the Skill Test Scoring System}

We assessed interrater reliability of the skill test scoring system with intraclass correlation coefficient (ICC) using all skill test scores (pre-, immediate post-, and 8-week post-training).

\section{Sample Size and Power Calculation}

Sample size calculation was based on our pilot study with 6 pre-clinical medical students [20]. The pilot study showed that the mean skill test score difference between pre-training and 8-week post-training was 2.28 points [standard deviation (SD), 4.44]. Using this estimate, 25 participants were required to provide $80 \%$ power with a one-sided alpha level of 0.05 . Assuming an approximately $15 \%$ participant withdrawal from the study, the sample size required was 29 . Participation in the study was voluntary; thus, sampling was not random. To increase representativeness of the sample and precision of the outcome measures, we continued to recruit participants for the planned study period even after the statistical sample size was achieved.

\section{Statistical Analysis}

All statistical analyses were performed using BellCurve for Excel (Social Survey Research Information Co., Ltd.). All numeric variables are presented as mean and SD, or median and interquartile range. Mean difference (MD) between pre- and 8-week post-training data was calculated with an unpaired t-test and 
presented as mean and SD with 95\% confidence interval $(\mathrm{Cl})$ and the ES. MD between pre- and immediate post-training data was calculated with a paired t-test and presented as mean and SD with $95 \% \mathrm{Cl}$ and the ES. We interpreted the clinical significance of ES according to Cohen's ES guidelines (ES of 0.2-0.5 = small ES, $0.5-0.8=$ moderate ES, and $>0.8=$ large ES) $[27,28]$. ICC estimates and their $95 \%$ Cls were calculated based on a mean rating $(k=3)$, absolute agreement, two-way random-effects model for interrater reliability and two-way mixed-effects model for test-retest reliability [29].

This manuscript adheres to the Guideline for Reporting Evidence-based practice Educational interventions and Teaching (GREET) with the GREET checklist (Additional File 11) [30].

\section{Results}

\section{Early Termination of Study Due to COVID-19 Restrictions}

In March of 2020, due to the coronavirus disease 2019 (COVID-19) pandemic, the University of Hawaii Human Studies Program recommended pausing research that involved any face-to-face interaction until after the crisis abated for researcher and study participant safety. In response to the recommendation, we terminated our study on March 16, 2020.

\section{Participant Characteristics}

Of the 149 eligible students at JABSOM, 54 participated in the study. All 54 students completed the pretraining assessment, hands-on training session, and immediate post-training assessment. Twenty-seven students (50\%) completed the 8-week post-training assessment, whereas the remaining 27 (50\%) did not because of early study termination (Fig. 3). Student characteristics and cardiac ultrasound training experience are in Table 2. Subgroup characteristics of students with all the tests completed $(n=27)$ and without 8-week post-training tests completed $(n=27)$ are also in Table 2. No students received structured ultrasound hands-on training or lecture before study participation. 
Table 2

Participant characteristics with subgroup characteristics of students with all tests completed and without 8-week post-training tests completed

\begin{tabular}{|c|c|c|c|}
\hline & $\begin{array}{l}\text { Students } \\
\text { with all } \\
\text { tests } \\
\text { completed } \\
(n=27)\end{array}$ & $\begin{array}{l}\text { Students without 8- } \\
\text { week post-training } \\
\text { tests completed } \\
(n=27)\end{array}$ & $\begin{array}{l}\text { All } \\
\text { participating } \\
\text { students } \\
(n=54)\end{array}$ \\
\hline 1 st year/2nd year student & $\begin{array}{l}17(63) / 10 \\
(37)\end{array}$ & $10(37) / 17(63)$ & $\begin{array}{l}27(50) / 27 \\
(50)\end{array}$ \\
\hline Age (years) & $25.0 \pm 3.6$ & $25.1 \pm 2.5$ & $25.1 \pm 3.1$ \\
\hline Female & $10(37)$ & $17(63)$ & $27(50)$ \\
\hline Left hand dominant & $2(7)$ & $2(7)$ & $4(7)$ \\
\hline $\begin{array}{l}\text { Pre-training skill test score } \\
\text { (10-point maximum) }\end{array}$ & $3.20 \pm 1.48$ & $2.21 \pm 1.75$ & $2.70 \pm 1.68$ \\
\hline $\begin{array}{l}\text { Pre-training knowledge test score } \\
\text { (40-point maximum) }\end{array}$ & $17.1 \pm 9.5$ & $13.8 \pm 9.6$ & $15.4 \pm 9.6$ \\
\hline \multirow[t]{8}{*}{ First-choice residency program } & IM 6 (22) & IM 7 (26) & IM 13 (24) \\
\hline & GS 6 (22) & Peds 4 (15) & GS 9 (17) \\
\hline & EM 5 (19) & EM 3 (11) & EM 8 (15) \\
\hline & $\begin{array}{l}\text { Ortho } 4 \\
(15)\end{array}$ & GS $3(11)$ & Peds 7 (13) \\
\hline & \multirow{3}{*}{$\begin{array}{l}\text { Peds } 3(11) \\
\text { Others } 2 \\
(7)\end{array}$} & FM 3 (11) & FM 4 (7) \\
\hline & & OB/GYN 3 (11) & Ortho 4 (7) \\
\hline & & Others 3 (11) & $\begin{array}{l}\text { Others } 7 \\
\text { (13) }\end{array}$ \\
\hline & $\begin{array}{l}\text { Undecided } \\
1(4)\end{array}$ & Undecided 1 (4) & $\begin{array}{l}\text { Undecided } 2 \\
(4)\end{array}$ \\
\hline \multicolumn{4}{|l|}{ Previous ultrasound training experience } \\
\hline $\begin{array}{l}\text { Structured ultrasound hands-on training or } \\
\text { lecture }\end{array}$ & $0(0)$ & $0(0)$ & $0(0)$ \\
\hline $\begin{array}{l}\text { Unstructured ultrasound hands-on training or } \\
\text { lecture }\end{array}$ & $15(56)$ & $8(30)$ & $23(43)$ \\
\hline Cardiac ultrasound on patients & $1(4)$ & $0(0)$ & $1(2)$ \\
\hline $\begin{array}{l}\text { Observation of a cardiac ultrasound on } \\
\text { patients }\end{array}$ & $16(59)$ & $14(52)$ & $30(56)$ \\
\hline
\end{tabular}




\begin{tabular}{|c|c|c|c|}
\hline & $\begin{array}{l}\text { Students } \\
\text { with all } \\
\text { tests } \\
\text { completed } \\
(n=27)\end{array}$ & $\begin{array}{l}\text { Students without 8- } \\
\text { week post-training } \\
\text { tests completed } \\
(n=27)\end{array}$ & $\begin{array}{l}\text { All } \\
\text { participating } \\
\text { students } \\
(n=54)\end{array}$ \\
\hline Cardiac ultrasound on healthy volunteers & $21(78)$ & $13(48)$ & $34(63)$ \\
\hline Cardiac ultrasound on simulators & $2(7)$ & $1(4)$ & $3(6)$ \\
\hline Experience using HHU (Butterfly iQ) & $4(15)$ & $4(15)$ & $8(15)$ \\
\hline $\begin{array}{l}\text { Completion of pre-training self-study of the ASE } \\
\text { online module }\end{array}$ & $27(100)$ & $26(96)$ & $53(98)$ \\
\hline $\begin{array}{l}\text { ASE online module review between immediate } \\
\text { post-training tests and 8-week post-training } \\
\text { tests }\end{array}$ & $6(22)$ & NA & NA \\
\hline $\begin{array}{l}\text { Review of textbooks or websites other than the } \\
\text { ASE module between immediate post-training } \\
\text { tests and 8-week post-training tests }\end{array}$ & $11(41)$ & NA & NA \\
\hline $\begin{array}{l}\text { Additional hands-on training between } \\
\text { immediate post-training tests and 8-week post- } \\
\text { training tests }\end{array}$ & $0(0)$ & NA & NA \\
\hline \multicolumn{4}{|c|}{$\begin{array}{l}\text { ASE, American Society of Echocardiography; } E M \text {, emergency medicine; } F M \text {, family medicine; } G S \text {, } \\
\text { general surgery; IM, internal medicine; IR, interventional radiology; } N A \text {, not applicable; Ortho, } \\
\text { orthopedics; Peds, pediatrics. }\end{array}$} \\
\hline Data are presented as mean \pm SD or $n(\%)$. & & & \\
\hline
\end{tabular}

\section{Outcome Measures}

Mean skill and knowledge test scores with median and individual scores are shown in Fig. 4. Breakdown of skill test scores for the 5 views are summarized in Table 3 . 
Table 3

Mean skill test scores and breakdown scores for 5 cardiac POCUS views

Pre-training Immediate post-training 8-week post-training

$\begin{array}{llll}\text { Skill test score }^{\star} & 2.70 \pm 1.68 & 7.91 \pm 1.12 & 4.82 \pm 2.28 \\ \text { PLAX score }^{\dagger} & 0.51 \pm 0.78 & 1.61 \pm 0.57 & 0.73 \pm 0.84 \\ \text { PSAX score }^{\dagger} & 0.49 \pm 0.63 & 1.69 \pm 0.39 & 0.52 \pm 0.79 \\ \text { A4C score }^{\dagger} & 0.32 \pm 0.47 & 1.11 \pm 0.49 & 0.68 \pm 0.65 \\ \text { S4C score }^{\dagger} & 1.06 \pm 0.73 & 1.54 \pm 0.47 & 1.40 \pm 0.61 \\ \text { SIVC score }^{\dagger} & 0.33 \pm 0.72 & 1.97 \pm 0.12 & 1.49 \pm 0.83\end{array}$

$A 4 C$, apical 4-chamber view; PLAX, parasternal long-axis view; PSAX, papillary muscle level of parasternal short-axis view; $S I V C$, subcostal inferior vena cava view; $S 4 C$, subcostal 4-chamber view.

Data are presented as mean \pm SD.

*10-point maximum score, ${ }^{\dagger} 2$-point maximum score.

The 2-point maximum scores for each of the 5 cardiac POCUS views are added for the 10-point maximum skill test score.

\section{Skill test score improvement}

[ ] The skill test score difference between pre-training and 8-week post-training was 2.11 points $(95 \% \mathrm{Cl}$, 1.22-3.00; large ES of 1.13), and [ ] the difference between pre-training and immediate post-training was 5.20 points $(95 \% \mathrm{Cl}, 4.71-5.70$; large ES of 3.68$)$.

\section{Knowledge test score improvement}

[ ] The knowledge test score difference between pre-training and 8-week post-training was 19.6 points ( $95 \% \mathrm{Cl}, 15.4-23.8$; large ES of 2.24), and [ ] the difference between pre-training and immediate posttraining was 23.1 points $(95 \% \mathrm{Cl}, 20.5-25.6$; large ES of 3.30).

\section{Post-training questionnaire}

The mean 5-point Likert rating of [ ] overall curriculum satisfaction were $4.9 \pm 0.6$ and $4.8 \pm 0.4$, [ ] the ASE online module satisfaction were $3.9 \pm 0.6$ and $4.1 \pm 0.6$, and [ ] hands-on training satisfaction were $4.9 \pm 0.6$ and $5.0 \pm 0.2$ at immediate post- and 8-week post-training, respectively (mean \pm SD). The mean 5-point Likert ratings of [ ] student motivation to purchase a personal HHU were $3.4 \pm 0.9,4.0 \pm 0.8$, and $4.0 \pm 0.9$ at pre-, immediate post-, and 8-week post-training, respectively (mean \pm SD).

\section{Subgroup analysis}


We found significant skill test score variation at 8-week post-training by visual inspection (Fig. 4A). Subgroup characteristics of students with a skill test score of 5 points or higher and less than 5 points at 8-week post-training are in Additional File 12. Compared to students with less than 5 points, students with

5 points or higher tended to have higher scores on pre-training knowledge tests, include more males, and first-year students. All students in both subgroups received no additional hands-on training between immediate post- and 8-week post-training tests. Because of the small subgroup sample size, we could not reach any conclusions about individual skill retention variation.

\section{Interrater Reliability of the Skill Test Scoring System}

Interrater reliability of the skill test scoring system assessed using all 135 score results of 10-point maximum skill tests from the 54 students was excellent (ICC, $0.93 ; 95 \% \mathrm{Cl}, 0.76-0.97$ ).

\section{Discussion}

The ASE-recommended cardiac POCUS curriculum demonstrated medium-term retention, not only shortterm, of cardiac POCUS image acquisition skills. This study is the first to provide learning effect evidence on longevity and durability of learned skill from a cardiac POCUS curriculum in a scientifically robust method, including collecting validity evidence for scoring systems. The curriculum was developed with the ASE medical education framework and linked with competency evaluation. It can be utilized as a standardized introductory cardiac POCUS curriculum for medical students or other novices. With reference to our study findings, educators can develop and institute effective and efficient curricula at their schools.

\section{Previous Research}

The 8 weeks post-training timeframe for retention assessment in our study was chosen based on 2 previous studies [31,32]. Fisher et al. studied skill retention after cadaver training for pigtail thoracostomy, femoral line placement, and endotracheal intubation with medical students, and reported that improved skills declined between 6 and 12 weeks [31]. Fisher et al. concluded that a refresher course should be considered when teaching complex technical skills. The study findings were consistent with skill degradation in 3 views (PLAX, PSAX, and A4C) in our study. Rappaport et al. investigated medical student temporal degradation of image acquisition skill 1, 4, and 8 weeks after cardiac, lung, and vascular ultrasound training [32]. Skill decay occurred at 8 weeks for PLAX and at 4 weeks for PSAX and SIVC, whereas lung, and vascular ultrasound skills did not decline statistically. Rappaport et al. assumed that the skill decay for cardiac images was due to the relatively higher complexity of the image acquisition compared with simpler pleural and vascular image acquisition. Interestingly, medical students in Rappaport's study experienced SIVC skill degradation, which did not occur in our study. A possible reason for the conflicting results is that simultaneous integration of 3 different ultrasound trainings in a single curriculum may have imposed a cognitive load on the novice learners that surpassed their memory capacity [33]. Alternatively, our blended-learning curriculum with the ASE framework may have 
contributed to the retention difference. Rappaport's instructional design included a 1-hour lecture and a 1hour supervised hands-on training without a pre-training self-study. Rappaport did not report concepts of curriculum design, detailed teaching methods, and validity evidence of assessment tools, which were essential to an effective curriculum development for skill retention [21]; these were reported in our study.

\section{Future Echocardiography Training in Medical School}

During the COVID-19 pandemic, medical schools worldwide have been facing unprecedented challenges in education delivery. Medical schools are limiting ward-based teaching and shifting their teaching format from face-to-face to online. To address this rapidly changing educational environment, development of standardized online-based teaching with scientific validation is urgently needed for all medical students who are missing their usual, previously planned education. Several medical students who were scheduled to participate in our cardiac POCUS curriculum missed additional hands-on practice because of early study termination due to COVID-19 restrictions. Future echocardiography training in medical school should be online- and/or simulation-based education that minimizes face-to-face and bedside teaching for both student and patient safety [34]. The ASE recommends 3 core components of cardiac POCUS education; didactic education, hands-on training, and image interpretation [7]. Didactic education and image interpretation teaching can be provided entirely online. With regard to hands-on training, instructorled face-to-face teaching with real-time feedback is still needed for image acquisition skill development $[9,35]$. In our study, we utilized the ASE online module to minimize lecture time and maximize hands-on practice time on the hands-on training day. However, a face-to-face instruction on 5-view image acquisition for $10 \mathrm{~min}$, which could be provided online, was still needed. This is because the ASE online module did not provide a detailed instruction on the item. If the ASE online module included the instruction, our lecture session could be shortened by $10 \mathrm{~min}$. We encourage undergraduate medical education program directors to utilize the ASE module with our video instruction on 5-view image acquisition to develop more comprehensive online-based curricula in the future.

\section{Limitations}

First, the primary outcome may represent attrition bias because 27 participants did not complete the 8week post-training assessment. However, the dropout was not caused by participant factors but COVID19 restrictions, which was unavoidable for both researchers and participants. Therefore, the dropout was unlikely to cause systematic differences between the 27 participants who followed up at 8 weeks and the 27 did not; the primary outcome result may be considered representative of all 54 participants.

Second, breakdown of the skill test scores showed that PLAX, PSAX, and A4C scores at 8-week posttraining did not achieve 1.0 point, indicating poor or unacceptable quality for clinical use. One plausible reason for this is the additional difficulty of obtaining those 3 views. Parasternal and apical views (PLAX, PSAX, and A4C) require probe placement in the intercostal space avoiding the lung and ribs with careful

probe manipulation. Compared with these views, subcostal views (S4C and SIVC) are obtained by placing the probe under the xiphoid process without fine probe manipulation in the intercostal space. Subcostal views were therefore relatively easy to obtain, required less precise probe manipulation, and may have 
been easier techniques to recall. Parasternal and apical views were in contrast challenging to obtain and may have been difficult for novice students to recall the technique. To develop more effective curricula for retaining skills, instructional design modifications or refresher training of the parasternal and apical views are needed.

Third, this study did not include a control group. Therefore, our curriculum did not demonstrate its superiority over other curricula. When developing the study protocol, we considered designing comparative study with a control group. However, neither a standardized curriculum nor curriculum with medium- or long-term skill retention effects was available for comparison. Thus, we addressed the development of a standardized curriculum that could demonstrate lasting educational benefits using the ASE curriculum framework. Future comparative studies are encouraged to utilize our curriculum as a control group for more effective and practical curriculum development.

Fourth, the skill test scoring system evaluated image acquisition skills to adjust probe position but did not assess image optimization skills to adjust ultrasound imaging parameters, patient position, or controlling patient breathing, because these adjustments were preset by the study protocol. Therefore, whether the students were able to obtain similar quality images to the study results without the study presetting is unknown. When integrating cardiac POCUS curricula into medical schools, workflow training incorporating these adjustments is warranted for curriculum comprehensiveness and practicality.

Fifth, the students in this study were volunteer participants, who may have been highly-motivated students to learn cardiac POCUS. Therefore, student skill retention might in this study be overestimated [36].

Finally, we only investigated medium-term retention 8 weeks after initial training; we did not explore longer term retention after more than 1 year. Future studies that follow up the durability of acquired skills for at least 1 year are warranted to develop a curriculum with long-term skill retention.

\section{Conclusions}

Our cardiac POCUS blended-learning curriculum with the ASE framework demonstrated medium-term retention of image acquisition skills 8 weeks after the initial training with a large ES of skill test score improvements. Educators can utilize the curriculum design as a reference while developing standardized curricula efficient for both trainees and trainers. However, breakdown of skill test scores showed that the image quality of PLAX, PSAX, and A4C at 8-week post-training was not acceptable for clinical use. Therefore, instructional design modifications of the 3 views or refresher training are needed to make this curriculum more effective for clinically relevant skill retention.

\section{Abbreviations}

ASE

American Society of Echocardiography 
A4C

Apical 4-chamber view

$\mathrm{Cl}$

Confidence interval

COVID-19

Coronavirus disease 2019

$\mathrm{HHU}$

Handheld ultrasound

ICC

Intraclass correlation coefficient

JABSOM

John A. Burns School of Medicine

MD

Mean difference

PLAX

Parasternal long-axis view

POCUS

Point-of-care ultrasound

PSAX

Papillary muscle level of parasternal short-axis view

SIVC

Subcostal inferior vena cava view

SD

Standard deviation

S4C

Subcostal 4-chamber view.

\section{Declarations}

\section{Acknowledgments}

We appreciate Dr. Kentaro Jujo (Department of Cardiology, Tokyo Women's Medical University Medical Center East, Japan) and the SUNRISE Lab (http://sunrise-lab.net/) for providing cardiologists' opinion. We are grateful to Dr. Natsinee Athinartrattanapong for providing advice on cardiac POCUS curriculum development. We are also thankful to Dr. Yuka Eto (SimTiki Simulation Center, John A. Burns School of Medicine, University of Hawaii at Manoa, HI, USA) for the assistance during the hands-on training. We are grateful to Dr. Atsushi Shiraishi, PhD (Emergency and Trauma Center, Kameda Medical Center, Chiba, Japan) and Dr. Hiroki Matsui (Clinical Research Unit, Kameda Medical Hospital/Kameda College University of Health Sciences, Chiba, Japan) for providing advice on test scoring system validation and primary outcome selection. We are thankful to Dr. Yoshihito Otsuka, MT, PhD (Clinical Laboratory Administration Department, Kameda General Hospital, Chiba, Japan) and sonographers in Kameda 
General Hospital that they gave us permission to tour their echocardiography laboratory and kindly share sonographer TTE teaching methods.

\section{Authors' contributions}

SJ conceived and developed the whole curriculum design, performed the data analysis, created the figures and tables, and prepared the manuscript. BS recruited participants, took a role of an assistant in the hands-on training, provided advice from a medical student perspective, and prepared the manuscript. JJL supervised the study, developed the curriculum design, recruited participants, took a role of an assistant in the hands-on training, performed the data analysis, and prepared the manuscript. $\mathrm{AK}, \mathrm{MI}$, and $\mathrm{KK}$ helped with the development of curriculum and the test scoring systems, hands-on training methodology, blinded rater assessment, and preparing the manuscript. AN helped with the development of curriculum and the knowledge test scoring system, took a role of an assistant in the hands-on training, and prepared the manuscript. SO provided critical advice from an educationalist perspective and prepared the manuscript. YK provided advice on statistics and an educational research design, and prepared the manuscript. BWB supervised the study, developed the curriculum design, recruited participants, took a role of an assistant in the hands-on training, performed the data analysis, and prepared the manuscript.

\section{Availability of data and materials}

The datasets used and/or analyzed during the current study are available from the corresponding author on reasonable request.

\section{Ethics approval and consent to participate}

The study was approved by the University of Hawaii Human Studies Program (Protocol number: 201900265).All study participants provided informed consent and all data were de-identified after collection.

\section{Consent for publication}

Not applicable.

\section{Competing interests}

The authors report no conflicts of interest.

\section{Funding}

None

\section{References}


1. Breitkreutz R, Price S, Steiger HV, Seeger FH, Ilper H, Ackermann H, et al. Focused echocardiographic evaluation in life support and peri-resuscitation of emergency patients: a prospective trial. Resuscitation. 2010;81:1527-33.

2. Via G, Hussain A, Wells $M$, Reardon R, ElBarbary M, Noble VE, et al. International evidence-based recommendations for focused cardiac ultrasound. J Am Soc Echocardiogr. 2014;27:683.e1-683.e33.

3. Schnobrich DJ, Mathews BK, Trappey BE, Muthyala BK, Olson APJ. Entrusting internal medicine residents to use point of care ultrasound: Towards improved assessment and supervision. Med Teach. 2018;40:1130-5.

4. Smith A, Parsons M, Renouf T, Boyd S, Rogers P. A mixed-methods evaluation of a multidisciplinary point of care ultrasound program. Med Teach. 2019;41:223-8.

5. Kirkpatrick JN, Grimm R, Johri AM, Kimura BJ, Kort S, Labovitz AJ, et al. Recommendations for echocardiography laboratories participating in cardiac point of care cardiac ultrasound (POCUS) and critical care echocardiography training: report from the American Society of Echocardiography. J Am Soc Echocardiogr. 2020;33:409-22.e4.

6. Seetharam K, Kagiyama N, Sengupta PP. Application of mobile health, telemedicine and artificial intelligence to echocardiography. Echo Res Pract. 2019;6:R41-52.

7. Johri AM, Durbin J, Newbigging J, Tanzola R, Chow R, De S, et al. Cardiac point-of-care ultrasound: state-of-the-art in medical school education. J Am Soc Echocardiogr. 2018;31:749-60.

8. European Society of Radiology. ESR statement on portable ultrasound devices. Insights Imaging. 2019;10:89.

9. Cawthorn TR, Nickel C, O'Reilly M, Kafka H, Tam JW, Jackson LC, et al. Development and evaluation of methodologies for teaching focused cardiac ultrasound skills to medical students. J Am Soc Echocardiogr. 2014;27:302-9.

10. Kusunose K, Yamada H, Suzukawa R, Hirata Y, Yamao M, Ise T, et al. Effects of transthoracic echocardiographic simulator training on performance and satisfaction in medical students. J Am Soc Echocardiogr. 2016;29:375-7.

11. Chaptal M, Tendron L, Claret P-G, Muller L, Markarian T, Mattatia L, et al. Focused cardiac ultrasound: a prospective randomized study of simulator-based training. J Am Soc Echocardiogr. 2020;33:4046.

12. Andersen GN, Viset A, Mjølstad OC, Salvesen O, Dalen H, Haugen BO. Feasibility and accuracy of point-of-care pocket-size ultrasonography performed by medical students. BMC Med Educ. 2014;14:156.

13. Ho AMH, Critchley LAH, Leung JYC, Kan PKY, Au SS, Ng SK, et al. Introducing final-year medical students to pocket-sized ultrasound imaging: teaching transthoracic echocardiography on a 2-week anesthesia rotation. Teach Learn Med. 2015;27:307-13.

14. Nelson BP, Hojsak J, Dei Rossi E, Karani R, Narula J. Seeing is believing: evaluating a point-of-care ultrasound curriculum for 1st-year medical students. Teach Learn Med. 2017;29:85-92. 
15. Kobal SL, Lior Y, Ben-Sasson A, Liel-Cohen N, Galante O, Fuchs L. The feasibility and efficacy of implementing a focused cardiac ultrasound course into a medical school curriculum. BMC Med Educ. 2017;17:94.

16. Neelankavil J, Howard-Quijano K, Hsieh TC, Ramsingh D, Scovotti JC, Chua JH, et al. Transthoracic echocardiography simulation is an efficient method to train anesthesiologists in basic transthoracic echocardiography skills. Anesth Analg. 2012;115:1042-51.

17. Fuchs L, Gilad D, Mizrakli Y, Sadeh R, Galante O, Kobal S. Self-learning of point-of-care cardiac ultrasound - Can medical students teach themselves? PLoS ONE. 2018;13:e0204087.

18. Kumar A, Barman N, Lurie J, He H, Goldman M, McCullough SA. Development of a point-of-care cardiovascular ultrasound program for preclinical medical students. J Am Soc Echocardiogr. 2018;31:1064-6.e2.

19. Kline J, Golinski M, Selai B, Horsch J, Hornbaker K. The effectiveness of a blended POCUS curriculum on achieving basic focused bedside transthoracic echocardiography (TTE) proficiency. A formalized pilot study. Cardiovasc Ultrasound. 2021;19:39.

20. Jujo S, Lee-Jayaram JJ, Sakka BI, Nakahira A, Kataoka A, Izumo M, et al. Pre-clinical medical student cardiac point-of-care ultrasound curriculum based on the American Society of Echocardiography recommendations: a pilot and feasibility study. Pilot feasibility Stud. 2021;7:175.

21. Motola I, Devine LA, Chung HS, Sullivan JE, Issenberg SB. Simulation in healthcare education: a best evidence practical guide. AMEE Guide No 82 Med Teach. 2013;35:e1511-30.

22. Neskovic AN, Skinner H, Price S, Via G, De Hert S, Stankovic I, et al. Focus cardiac ultrasound core curriculum and core syllabus of the European Association of Cardiovascular Imaging. Eur Heart $\mathrm{J}$ Cardiovasc Imaging. 2018;19:475-81.

23. Mitchell C, Rahko PS, Blauwet LA, Canaday B, Finstuen JA, Foster MC, et al. Guidelines for performing a comprehensive transthoracic echocardiographic examination in adults: recommendations from the American Society of Echocardiography. J Am Soc Echocardiogr. 2019;32:1-64.

24. Soni NJ, Arntfield R, Pierre K. Point-of-Care Ultrasound. 1st ed. Philadelphia: Saunders; 2014.

25. Butterfly Network Inc. Butterfly iQ User manual [Internet]. 2019 [cited 2020 May 25]. Available from: https://manual.butterflynetwork.com/butterfly-iq-user-manual_rev-s-en.pdf.

26. Edrich T, Seethala RR, Olenchock BA, Mizuguchi AK, Rivero JM, Beutler SS, et al. Providing initial transthoracic echocardiography training for anesthesiologists: simulator training is not inferior to live training. J Cardiothorac Vasc Anesth. 2014;28:49-53.

27. Cohen J. Statistical Power Analysis for the Behavioral Sciences. 2nd ed. Hillsdale: Lawrence Erlbaum; 1988.

28. Faraone SV. Interpreting estimates of treatment effects: implications for managed care. P T. 2008;33:700-11.

29. Koo TK, Li MY. A guideline of selecting and reporting intraclass correlation coefficients for reliability research. J Chiropr Med. 2016;15:155-63. 
30. Phillips AC, Lewis LK, McEvoy MP, Galipeau J, Glasziou P, Moher D, et al. Development and validation of the guideline for reporting evidence-based practice educational interventions and teaching (GREET). BMC Med Educ. 2016;16:237.

31. Fisher J, Viscusi R, Ratesic A, Johnstone C, Kelley R, Tegethoff AM, et al. Clinical skills temporal degradation assessment in undergraduate medical education. J Adv Med Educ Prof. 2018;6:1-5.

32. Rappaport CA, McConomy BC, Arnold NR, Vose AT, Schmidt GA, Nassar B. A prospective analysis of motor and cognitive skill retention in novice learners of point of care ultrasound. Crit Care Med. 2019;47:e948-52.

33. Young JQ, Van Merrienboer J, Durning S, Ten Cate O. Cognitive Load Theory: implications for medical education: AMEE Guide No. 86. Med Teach. 2014;36:371-84.

34. Johri AM, Galen B, James N, Lanspa M, Mulvagh S, Thamman R, et al. ASE Statement on Point-ofCare Ultrasound (POCUS) During the 2019 Novel Coronavirus Pandemic. 2020;1-8.

35. Kimura BJ, Sliman SM, Waalen J, Amundson SA, Shaw DJ. Retention of Ultrasound Skills and Training in "Point-of-Care" Cardiac Ultrasound. J Am Soc Echocardiogr. 2016;29:992-7.

36. Callahan CA, Hojat M, Gonnella JS. Volunteer bias in medical education research: an empirical study of over three decades of longitudinal data. Med Educ. 2007;41:746-53.

\section{Figures}

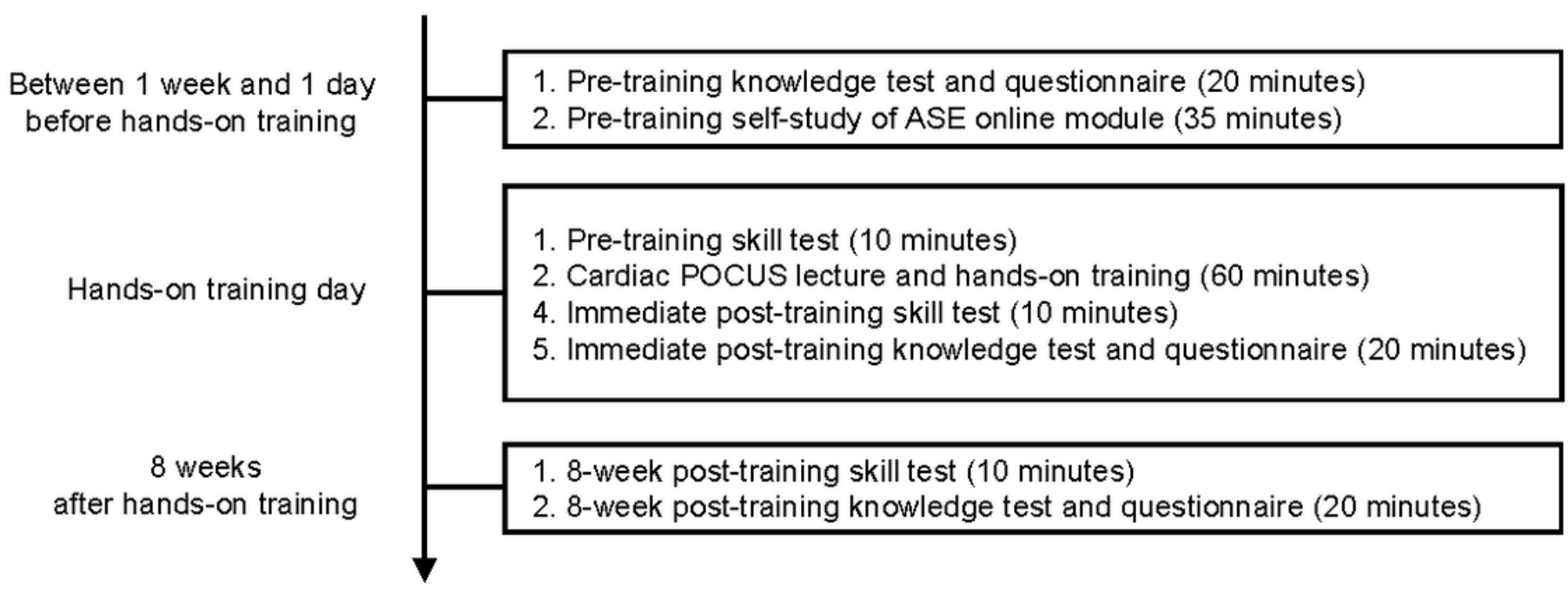

\section{Figure 1}

Cardiac point-of-care ultrasound curriculum timeline. ASE, American Society of Echocardiography; POCUS, point-of-care ultrasound. 


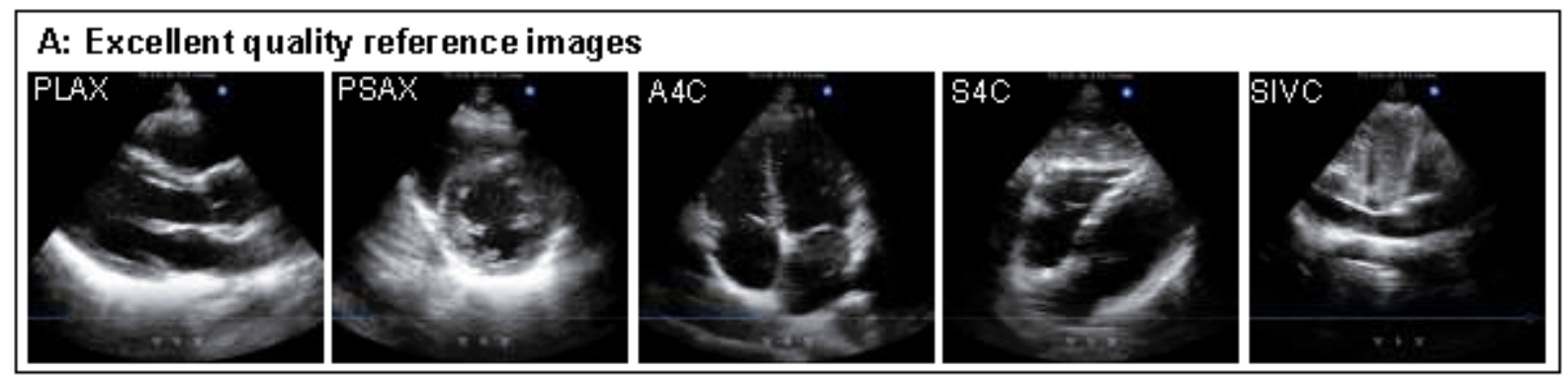

\section{B: Acceptable quality images}
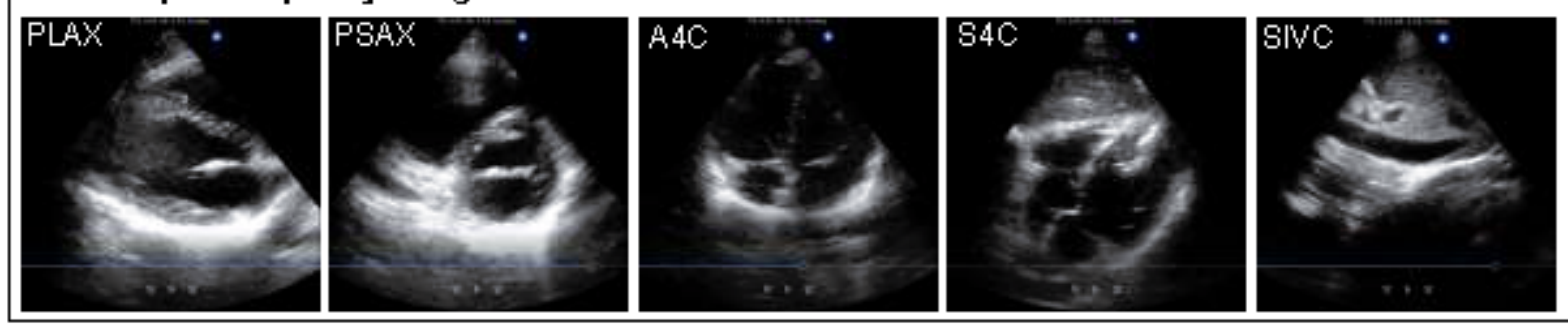

\section{C: Poor quality images}
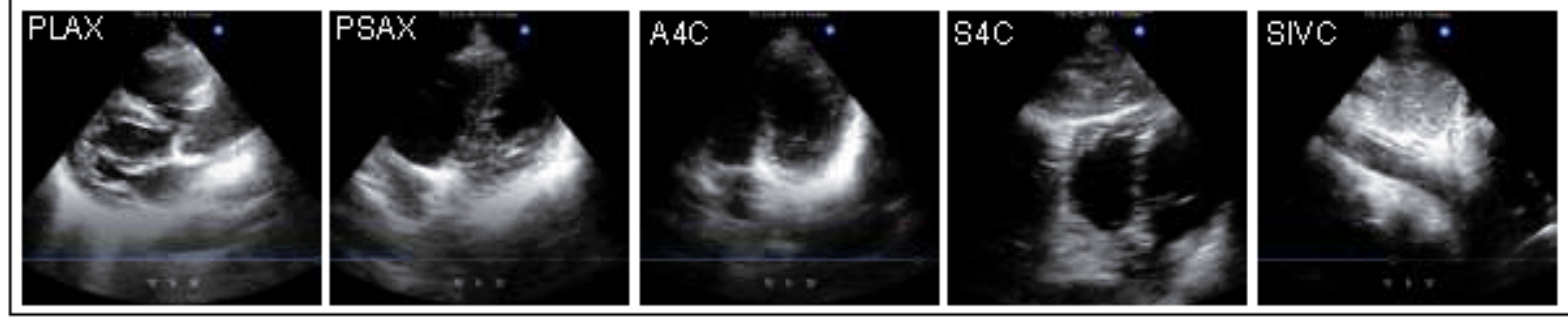

\section{Figure 2}

Excellent quality reference (A) and examples of acceptable (B) and poor quality (C) images of 5 cardiac POCUS views. Excellent quality reference images $(\mathbf{A})$ refer to 5 cardiac POCUS views obtained by the cardiologist (MI) on the healthy volunteer (SJ) used for all skill tests. Examples of acceptable (B) and poor quality (C) images refer to the 5 views obtained by medical students on the healthy volunteer. Adapted from Jujo et al [20]. A4C, apical 4-chamber view; PLAX, parasternal long-axis view; POCUS, pointof-care ultrasound; $P S A X$, papillary muscle level of parasternal short-axis view; $S I V C$, subcostal inferior vena cava view; $S 4 C$, subcostal 4-chamber view. 


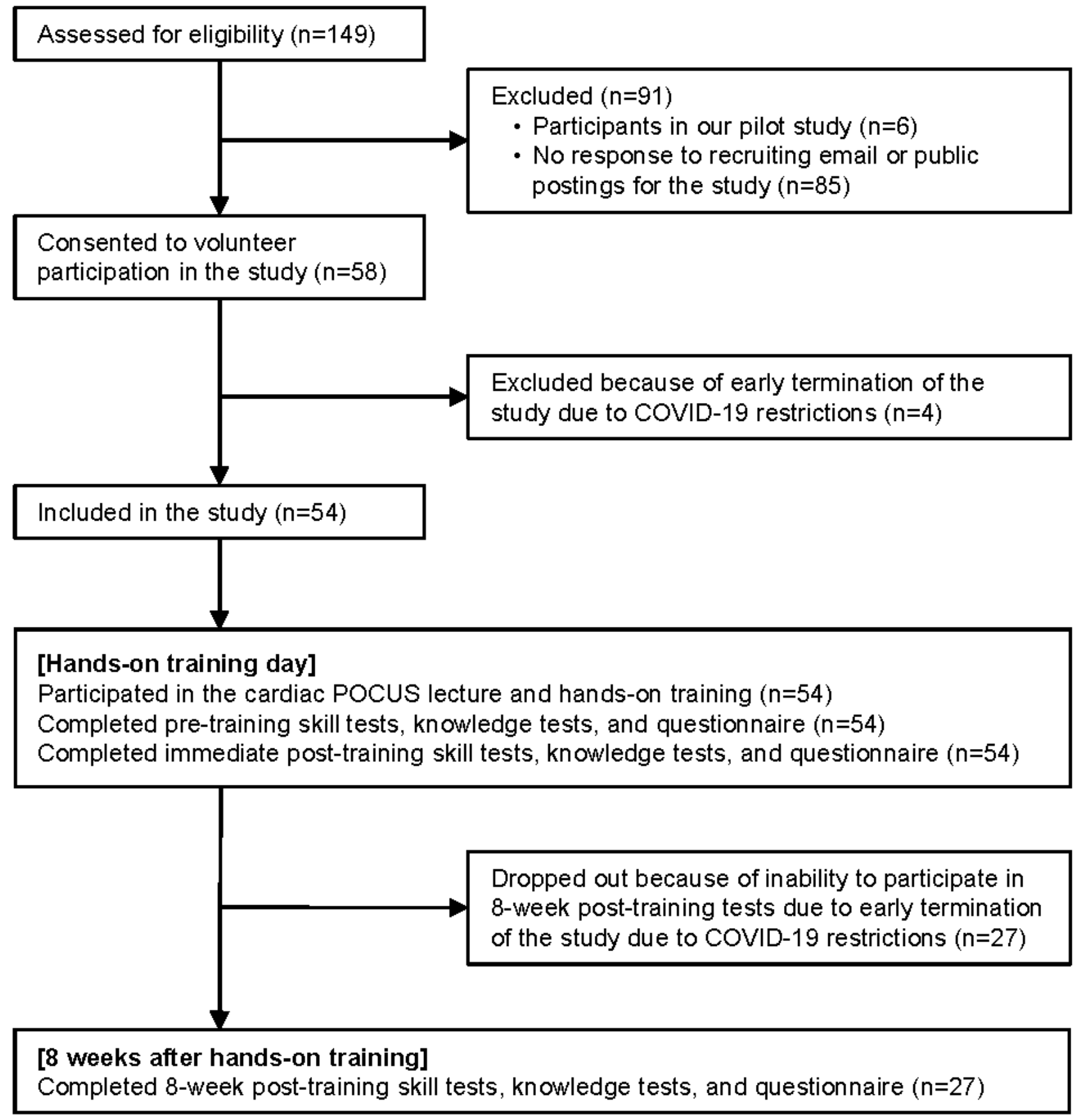

Figure 3

Study flow. 

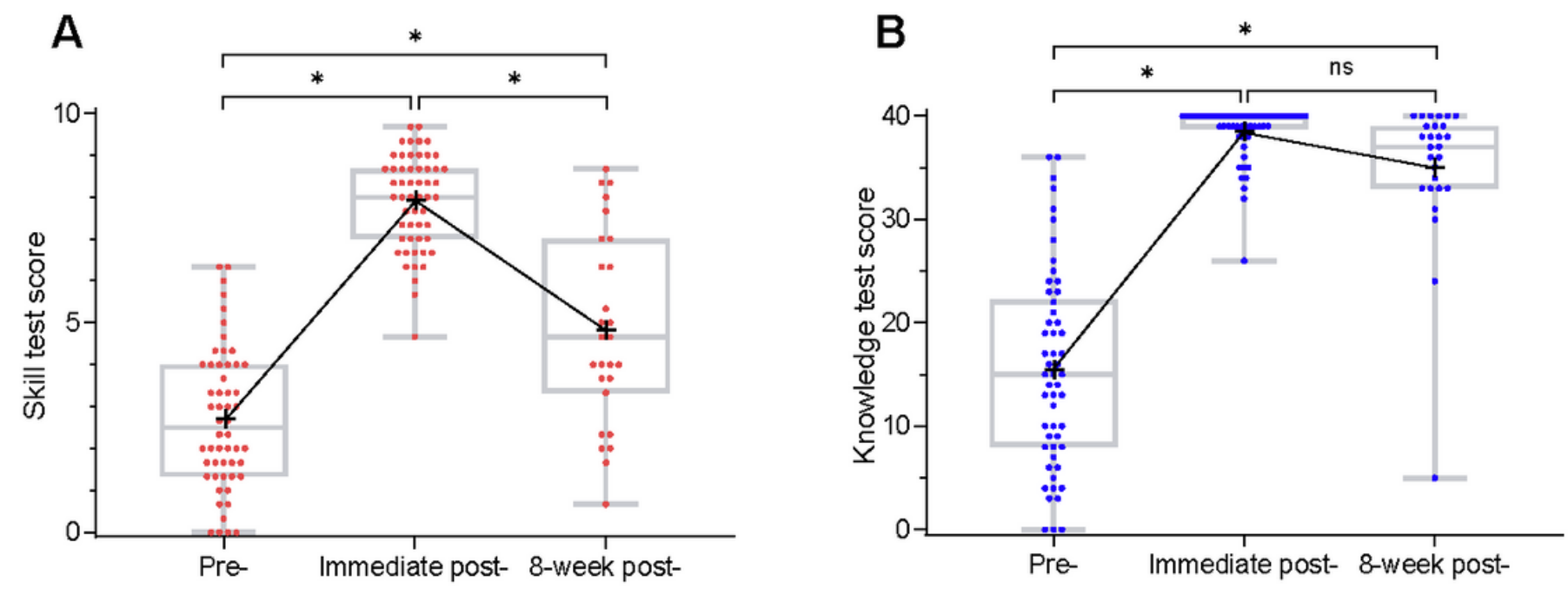

Figure 4

Mean skill (A) and knowledge (B) test scores with median and individual scores. Red ( $)$ and blue dots (๑) indicate individual scores. Boxplots indicate minimum, maximum, median, lower, and upper quartiles. Crosses (+) indicate mean. $n s$, not significant. $p<.0001$.

\section{Supplementary Files}

This is a list of supplementary files associated with this preprint. Click to download.

- AdditionalFile1ContentofPOCUSlecture.docx

- AdditionalFile10DiscriminatoryAbilityofknowledge.pptx

- AdditionalFile11GREETchecklist.docx

- AdditionalFile12Subgroupcharacteristics.docx

- AdditionalFile3Maininstructionpoints.docx

- AdditionalFile4VideoSkillTest.mp4

- AdditionalFile5VideoExcellent.mp4

- AdditionalFile6VideoAcceptable.mp4

- AdditionalFile7Videopoor.mp4

- AdditionalFile8DiscriminatoryAbilityofskill.pptx

- AdditionalFile9knowledgetest.pdf

- Additionalfile2Video5viewinstruction.mp4 